\title{
Towards Formal Verification on the System Level
}

(Invited Talk)

\author{
Rolf Drechsler \\ Institute of Computer Science \\ University of Bremen \\ 28359 Bremen, Germany \\ drechsle@informatik.uni-bremen.de
}

\begin{abstract}
Due to increasing design complexity and intensive reuse of components, verifying the correctness of circuits and systems becomes a more and more important factor. In the meantime, in many projects up to $80 \%$ of the overall design costs are caused by verification and by this, checking the correct behavior becomes the dominating factor.

Formal verification has been proposed as a promising alternative to simulation and has become a standard in many flows. In this paper, existing approaches are reviewed and recent trends for system level verification are outlined. To demonstrate the techniques SystemC is used as a system level description language.

Beside the successful applications a list of challenging problems is provided. This gives a better understanding of current problems in hardware verification and shows directions for future research.
\end{abstract}

\section{Introduction}

With increasing design complexity, verification becomes a more and more important aspect of the design flow. Modern circuits contain up to several hundred million transistors. In the meantime it has been observed that verification becomes the major bottleneck, i.e. up to $80 \%$ of the overall design costs are due to verification. This is one of the reasons why recently several methods have been proposed as alternatives to classical simulation, since it cannot guarantee sufficient coverage of the design. E.g. in [2] it has been reported that for the verification of the Pentium IV more than 200 billion cycles have been simulated, but this only corresponds to $2 \mathrm{CPU}$ minutes, if the chip is run with 1 $\mathrm{GHz}$.

Formal verification techniques have gained large attention, since they allow to prove the correctness of a circuit, i.e. they ensure $100 \%$ functional correctness. Beside be- ing more reliable, formal verification approaches have also shown to be more cost effective in many cases, since test bench creation - usually a very time consuming and error prone task - becomes superfluous [36].

In this paper, first briefly some of the application domains are described, where formal techniques have successfully been used. Some links to further literature are given where the interested reader can get more information. Then, aspects of system level verification are discussed, where SystemC is used as the modelling platform. Finally, a list of "challenging problems" is given, i.e. a list of topics that need further investigation in the context of formal hardware verification.

\section{Formal Verification}

The main idea of formal hardware verification is to prove the functional correctness of a design instead of simulating some vectors. For the proof process different techniques have been proposed. Most of them work in the Boolean domain, like Binary Decision Diagrams (BDDs) or SAT solvers.

The typical hardware verification scenarios where formal proof techniques are applied are

Equivalence Checking (EC) and

Property Checking (PC), also called Model Checking (MC).

The goal of EC is to ensure the equivalence of two given circuit descriptions. These circuits might be given on different levels of abstraction, i.e. register transfer level or gate level. The main steps of an equivalence checker are as follows (see e.g. [13]):

1. Translate both designs to an internal format.

2. Establish the correspondence between the two designs in a matching phase. 
3. Prove equivalence or inequivalence.

4. In case of an inequivalence a counter-example is generated and the debugging phase starts.

Notice that the circuit is considered as purely combinational by modeling the state elements as additional primary inputs and outputs. This modeling may result in counter-examples that are not reachable during normal circuit operation.

In contrast to EC, where two circuits are considered, for PC a single circuit is given and properties are formulated in a dedicated "verification language". It is then formally proven whether these properties hold under all circumstances. While "classical" CTL-based model checking [6] can only be applied to medium sized designs, approaches based on Bounded Model Checking (BMC) as discussed in [4] give very good results when used for complete blocks with up to $100 \mathrm{k}$ gates.

Nevertheless, all these approaches can run into problems caused by complexity, e.g. if the circuit becomes too large or if the function being represented turns out to be "difficult" for formal methods. The second problem often arises in cases of complex arithmetics, like multipliers.

Motivated by this, hybrid methods have been proposed, like e.g. symbolic simulation and assertion checking. These methods try to bridge the gap between simulation and correctness proofs. But these techniques also make use of formal proof techniques.

For more information on basics on formal verification techniques the reader is referred to [27].

\section{System Level Verification}

While classical approaches to circuit design make use of Hardware Description Languages (HDLs), like VHDL or Verilog, there is a strong interest in C-like description languages [19] for system level modelling. These languages allow for higher abstraction and fast simulation in an early stage of the design process. Furthermore, hardware/software co-design can be performed in the same system environment. One of the most popular languages of this type is SystemC [34] ${ }^{1}$. But so far, most verification approaches for SystemC are based on simulation only (see e.g. $[33,15])$. Of course, due to the reasons discussed in the introduction, it would be desirable to have formal verification techniques also at the system level.

Two approaches recently presented for verification of SystemC are briefly reviewed:

\section{Bounded Model Checking [17]}

\section{Generation of Checkers [18]}

For details on the approaches and experimental results we refer to the original papers.

\footnotetext{
${ }^{1}$ All techniques discussed in this section can also be transferred to other system level languages, like e.g. SystemVerilog.
}

\subsection{Bounded Model Checking}

In this section an approach to reason about the behavior of SystemC designs based on formally verifying properties specified in Linear Temporal Logic (LTL) is presented. The approach considers synchronous sequential circuits modelled in SystemC at the register transfer level. First, the output functions and transition functions of the underlying Finite State Machine (FSM) are computed. Then a symbolic reachability analysis of the FSM is carried out. Finally, proving an LTL formula is translated to a satisfiability problem using the transition and output functions and the set of reachable states. Unbounded LTL formulas can be proved, since the complete set of reachable states is known.

\subsection{Generation of Checkers}

There are several approaches to system level verification which are based on assertions [16]. The key idea is to describe expected or unexpected behavior directly in the device under test. These conditions are checked dynamically during simulation. In contrast in [10] a method has been proposed to synthesize properties for circuits into hardware checkers. Properties which have been specified for (formal) verification are directly mapped onto a very regular hardware layout.

Following the latter idea, a method is presented which allows checking of temporal properties for circuits and systems described in SystemC not only during simulation. A property is translated into a synthesizable SystemC checker and embedded into the circuit description. This enables the evaluation of the properties during simulation and after fabrication of the system. Of course, with this approach a property is not formally proven and only parts of the functionality are covered. But the proposed method is applicable to large circuits and systems and supports the checking of properties in form of an on-line test. This on-line test is applicable, even if formal approaches failed due to limited resources.

\section{Challenges}

Even though formal verification techniques are very successfully applied and have become the state-of-the-art in many design flows, still many problems exist. In this section a list of these problems is given. The list is not complete in the sense that all difficulties are covered, but many important ones are mentioned. This gives a better understanding of current problems in hardware verification and shows directions for future research.

Complexity: According to Moore's law the complexity of the circuits steadily increases. For this, the underlying data structures are very important. For EC and BMC 
often dedicated data structures are used. For representation of the state space BDDs have shown to work well, but if the size of the circuit becomes too large the BDDs often suffer from "memory explosion".

Proof technology: While BDDs and SAT are the most popular techniques in hardware verification and have also been applied to many domains, there is still a lot of research going on. Besides the classical monolithic approaches modern EC tools make use of multiengine approaches that combine different techniques, like SAT, BDD, term rewriting, ATPG, and random pattern simulation. How to successfully combine these - often orthogonal - approaches is not fully understood today.

Word-level approaches: Even though most proof techniques today work on the bit-level, many studies have shown that significant improvements can be achieved if the proof engine makes use of high-level information or even completely works on a higher level of abstraction. In this context also ILP solvers showed promise.

Matching in EC: As described above, before the proof process starts the correspondence between the circuits has to be established. Here, several techniques exist, like name-based, structural or prover-based, but still for large industrial designs these methods often fail. This results in very time consuming user defined matching.

Reachability of counter-examples: In EC and BMC the generated counter-example might not be reachable in normal circuit operation. This results from the modeling of the circuit, i.e. instead of a FSM only the combinational part is considered. Thus, it has to be checked that the counter-example is "valid" after it has been generated, or the prover has to ensure that it is reachable. Techniques have to be developed how this can be ensured without a complete reachability analysis of the FSM, that is usually not feasible due to complexity reasons.

Arithmetic: Industrial practice has shown that today's proof techniques, like BDD and SAT, have difficulties with arithmetic circuits, like multipliers. Word-level approaches have been proposed as an alternative, but these methods turned out to often be difficult to integrate in fully automatic tools. For this, arithmetic circuits - often occurring in circuit design - are still difficult to handle.

System integration: PC works best on the module level, i.e. for blocks with up to $100 \mathrm{k}$ gates. But in multichip modules many of these blocks are integrated to build a system. Due to complexity the modules cannot be verified as one large block and for this models and approaches are needed.
Hybrid approaches: For complex blocks or on the system level PC might be a very complex task and for this simpler alternatives have been studied, i.e. techniques that are more powerful than classical simulation but need less resources than PC. Techniques, like symbolic simulation or assertion-based verification, in this context also make use of formal verification techniques.

Checker synthesis: The specified properties can also be synthesized and added to the design. In this way, they can also be used for on-line test after the circuit has been fabricated.

Analog/mixed signal: Most EC and PC models assume that the circuit is purely digital, while in modern system-on-chip designs many analog components are integrated. For this, also models and proof mechanisms need to be developed for analog and mixed signal devices.

Retiming: For EC retimed circuits are still difficult to handle, since in this case the state matching cannot be performed. Thus, the problem remains sequential and by this becomes far too complex.

Multiple clocks: Many circuits have different clocking domains, while verification tools can often only work with a single clock.

Coverage: To check the completeness of a verification process coverage metrics have to be defined. While typical methods, like state coverage, are much too weak in the context of formal verification, there still does not exist a good measure that is comfortable to use for PC.

Diagnosis: After a fault has been identified by a formal verification tool a counter-example is generated. The next step is to identify the fault location or a reason for the failing proof process. Here, also formal proof techniques can be applied.

Most solutions to these problems are still in a very early stage of development, but these fields have to be addressed to make formal hardware verification successful in industrial applications. To orient the reader, some recent references are provided to give a starting point for further studies: $[30,22,27,21,9,31,14,1,7,28,26,20,5,24,29,25$, $32,12,23,35,3,16,10,8,11]$

\section{Conclusions}

In this paper formal verification with a special focus on system level verification has been discussed. While EC works very well on complete designs with several million transistors, PC approaches are so far mainly applicable at the block level. 
For a solution for complete systems, still many problems have to be solved, where some of the most important were given in the previous section.

In future design projects verification will become more and more important and the creation of a concise verification methodology decides about successful tape-outs.

\section{Acknowledgement}

The list of challenging problems has been developed in the context of the book project Advanced Formal Verification [11]. I like to thank all the contributors for the interesting discussions. Furthermore I like to thank Daniel Große for his contributions to the verification approaches for SystemC presented in Section 3.

\section{References}

[1] L. Bening and H. Foster. Principles of Verifiable RTL Design. Kluwer Academic Publishers, 2001.

[2] B. Bentley. Validating the Intel Pentium 4 microprocessor. In Design Automation Conf., pages 244-248, 2001.

[3] J. Bergeron. Writing Testbenches: Functional Verification of HDL Models. Kluwer Academic Publishers, 2003.

[4] A. Biere, A. Cimatti, E. Clarke, M. Fujita, and Y. Zhu. Symbolic model checking using SAT procedures instead of BDDs. In Design Automation Conf., pages 317-320, 1999.

[5] R. Brinkmann and R. Drechsler. RTL-datapath verification using integer linear programming. In ASP Design Automation Conf., pages 741-746, 2002.

[6] J. Burch, E. Clarke, K. McMillan, and D. Dill. Sequential circuit verification using symbolic model checking. In $D e$ sign Automation Conf., pages 46-51, 1990.

[7] H. Chockler, O. Kupferman, R. Kurshan, and M. Vardi. A practical approach to coverage in model checking. In Computer Aided Verification, volume 2102 of LNCS, pages 6677. Springer Verlag, 2001.

[8] F. Copty, A. Irron, O. Weissberg, N. Kropp, and G. Kamhi. Efficient debugging in a formal verification environment. Software Tools for Technology Transfer, 4:335-348, 2003.

[9] R. Drechsler. Formal Verification of Circuits. Kluwer Academic Publishers, 2000.

[10] R. Drechsler. Synthesizing checkers for on-line verification of system-on-chip designs. In IEEE International Symposium on Circuits and Systems, pages IV:748-IV:751, 2003.

[11] R. Drechsler. Advanced Formal Verification. Kluwer Academic Publishers, 2004.

[12] R. Drechsler and N. Drechsler. Evolutionary Algorithms for Embedded System Design. Kluwer Academic Publisher, 2002.

[13] R. Drechsler and S. Höreth. Gatecomp: Equivalence checking of digital circuits in an industrial environment. In Int'l Workshop on Boolean Problems, pages 195-200, 2002.

[14] R. Drechsler and D. Sieling. Binary decision diagrams in theory and practice. Software Tools for Technology Transfer, 3:112-136, 2001.

[15] F. Ferrandi, M. Rendine, and D. Scuito. Functional verification for SystemC descriptions using constraint solving. In Design, Automation and Test in Europe, pages 744-751, 2002.
[16] H. Foster, A. Krolnik, and D. Lacey. Assertion-Based Design. Kluwer Academic Publishers, 2003.

[17] D. Große and R. Drechsler. Formal verification of LTL formulas for SystemC designs. In IEEE International Symposium on Circuits and Systems, pages V:245-V:248, 2003.

[18] D. Große and R. Drechsler. Checkers for SystemC designs. In MEMOCODE, 2004.

[19] R. Gupta. IEEE design and test roundtable on C++-based design. IEEE Design \& Test of Comp., pages 115-123, 2001. May-June.

[20] S. Hassoun and T. Sasao. Logic Synthesis and Verification. Kluwer Academic Publishers, 2001.

[21] P.-H. Ho, T. Shiple, K. Harer, J. Kukula, R. Damiano, V. Bertacco, J. Taylor, and J. Long. Smart simulation using collaborative formal and simulation engines. In Int'l Conf. on $C A D$, pages $120-126,2000$.

[22] Y. Hoskote, T. Kam, P. Ho, and X. Zhao. Coverage estimation for symbolic model checking. In Design Automation Conf., pages 300-305, 1999.

[23] Y.-C. Hsu, B. Tabbara, Y.-A. Chen, and F. Tsai. Advanced techniques for RTL debugging. In Design Automation Conf., pages 362-367, 2003.

[24] P. Johannsen and R. Drechsler. Formal verification on register transfer level - utilizing high-level information for hardware verification. In IFIP Int'l Conf. on VLSI, pages 127132,2001

[25] R. Jones. Symbolic Simulation Methods for Industrial Formal Verification. Kluwer Academic Publishers, 2002.

[26] A. Kölbl, J. Kukula, and R. Damiano. Symbolic RTL simulation. In Design Automation Conf., pages 47-52, 2001.

[27] T. Kropf. Introduction to Formal Hardware Verification. Springer, 1999.

[28] A. Kuehlmann, M. Ganai, and V. Paruthi. Circuit-based Boolean reasoning. In Design Automation Conf., pages 232237, 2001.

[29] J. Mohnke, P. Molitor, and S. Malik. Limits of using signatures for permutation independent Boolean comparison. Formal Methods in System Design: An International Journal, 2(21):167-191, 2002.

[30] D. Moundanos, J. Abraham, and Y. Hoskote. Abstraction techniques for validation coverage analysis and test generation. IEEE Trans. on Comp., pages 2-14, January 1998.

[31] P. Rashinkar, P. Paterson, and L. Singh. System-on-a-Chip Verification. Kluwer Academic Publishers, 2000.

[32] S. Reda, R. Drechsler, and A. Orailoglu. On the relation between SAT and BDDs for equivalence checking. In Int'l Symp. on Quality Electronic Design, pages 394-399, 2002.

[33] J. Ruf, D. W. Hoffmann, T. Kropf, and W. Rosenstiel. Simulation-guided property checking based on multi-valued ar-automata. In Design, Automation and Test in Europe, pages 742-748, 2001.

[34] Synopsys Inc., CoWare Inc., and Frontier Design Inc., http://www.systemc.org. Functional Specification for SystemC 2.0.

[35] A. Veneris, A. Smith, and M. S. Abadir. Logic verification based on diagnosis techniques. In ASP Design Automation Conf., 2003.

[36] K. Winkelmann, H.-J. Trylus, D. Stoffel, and G. Fey. A cost-efficient block verification for a UMTS up-link chiprate coprocessor. In Design, Automation and Test in Europe, volume 1, pages 162-167, 2004. 